\title{
Emergency repair of inguinal hernia in the premature infant is associated with high direct medical costs
}

\author{
J. Verhelst ${ }^{1}$ - B. de Goede ${ }^{1}$ - B. J. H. van Kempen ${ }^{2}$ H. R. Langeveld ${ }^{3}$. \\ M. J. Poley ${ }^{3,4}$ - G. Kazemier ${ }^{5}$ J. Jeekel ${ }^{6}$ R. M. H. Wijnen ${ }^{3}$ - J. F. Lange ${ }^{1}$
}

Received: 20 March 2015/Accepted: 18 November 2015/Published online: 14 December 2015

(C) The Author(s) 2015. This article is published with open access at Springerlink.com

\begin{abstract}
Purpose Inguinal hernia repair is frequently performed in premature infants. Evidence on optimal management and timing of repair, as well as related medical costs is still lacking. The objective of this study was to determine the direct medical costs of inguinal hernia, distinguishing between premature infants who had to undergo an emergency procedure and those who underwent elective inguinal hernia repair.

Methods This cohort study based on medical records concerned premature infants with inguinal hernia who underwent surgical repair within 3 months after birth in a tertiary academic children's hospital between January 2010 and December 2013. Two groups were distinguished: patients with incarcerated inguinal hernia requiring
\end{abstract}

J. Verhelst and B. de Goede have contributed equally to this manuscript.

J. Verhelst

j.verhelst@erasmusmc.nl

1 Department of Surgery, Erasmus University Medical Center (Erasmus MC) Rotterdam, PO Box 2040,

3000 CA Rotterdam, The Netherlands

2 Department of Epidemiology and Radiology, Erasmus University Medical Center (Erasmus MC) Rotterdam, Rotterdam, The Netherlands

3 Department of Paediatric Surgery, Erasmus University Sophia Children's Hospital, Rotterdam, The Netherlands

4 Institute for Medical Technology Assessment (iMTA), Erasmus University Rotterdam, Rotterdam, The Netherlands

5 Department of Surgery, VU University Medical Center, Amsterdam, The Netherlands

6 Department of Neuroscience, Erasmus University Rotterdam, Rotterdam, The Netherlands emergency repair and patients who underwent elective repair. Real medical costs were calculated by multiplying the volumes of healthcare use with corresponding unit prices. Nonparametric bootstrap techniques were used to derive a $95 \%$ confidence interval (CI) for the difference in mean costs.

Results A total of 132 premature infants were included in the analysis. Emergency surgery was performed in $29 \%$. Costs of hospitalization comprised $65 \%$ of all costs. The total direct medical costs amounted to $€ 7418$ per premature infant in the emergency repair group versus $€ 4693$ in the elective repair group. Multivariate analysis showed a difference in costs of $€ 1183$ (95 \% CI -1196; 3044) in favor of elective repair after correction for potential risk factors. Conclusion Emergency repair of inguinal hernia in premature infants is more expensive than elective repair, even after correction for multiple confounders. This deserves to be taken into account in the debate on timing of inguinal hernia repair in premature infants.

Keywords Inguinal hernia $\cdot$ Premature infants $\cdot$ Cost analysis $\cdot$ Emergency repair

\section{Introduction}

Inguinal hernia repair is the most frequently performed procedure in pediatric surgery. The cumulative incidence of inguinal hernia is up to $9.0 \%$ in prematurely born children. More than half these patients will undergo hernia repair in the first year of life [1,2].

Eventually all premature infants with an inguinal hernia have to undergo surgical repair [3-5]. However, evidence on the optimal management and timing of inguinal hernia repair in these neonates is lacking. Delayed repair seems 
appealing as premature infants often have concomitant comorbidities and are vulnerable for perioperative complications [2]. On the other hand, delaying elective repair can be complicated by incarceration, in which case emergency surgery is needed. In a previous study we showed that more than $50 \%$ of premature infants with an inguinal hernia present with a symptomatic inguinal hernia, and emergency repair was needed in more than half of these cases due to incarceration [6]. Emergency repair is associated with a higher risk of surgical and anesthetic complications and has been found to result in a longer duration of hospitalization, more readmissions, and even reoperations $[2,6]$.

To prevent possible emergency repair in premature infants, some surgeons prefer to perform surgery during the birth hospitalization, before discharge from the neonatal intensive care unit (NICU). Although this intervention prolongs initial hospitalization, it avoids readmission for elective repair [7]. Delayed elective repair may also result in repeated herniation and reduction at the emergency department (ED), multiple visits to the outpatient clinic (OPC), and perhaps a longer perioperative admission on the pediatric ward or NICU, which is likely to raise direct medical costs $[8,9]$.

The timing of surgery is mostly based on expert opinion and the surgeon's personal preference. In the current era of tightening budgets, economic aspects have captured the interest of healthcare policy makers [10, 11]. Except for a mid-1980s study that compared treatment costs in premature infants operated on prior to discharge from the NICU and in full-term infants operated on as outpatients, there are no data on costs of inguinal hernia repair in premature infants in relation to timing of repair [7]. Bearing in mind that surgery in premature patients is very costly, insight into the actual costs of different strategies in inguinal hernia repair would be relevant for planning and organization of care, for supporting clinical decisions, and for efficient allocation of health care resources.

The objective of this study was to compare the medical costs of inguinal hernia repair in premature infants between patients who had to undergo emergency repair and those who underwent elective repair.

\section{Methods}

\section{Setting and patients}

A cohort study on medical records was performed at a tertiary academic children's hospital in the Netherlands. All premature infants (defined as gestational age $<37$ weeks) who presented at the ED or at the OPC and operated on for inguinal hernia within 3 months after birth between January 2010 and December 2013 were included. They were identified from the electronic hospital data systems and medical charts using the so-called "Centraal Orgaan Tarieven Gezondheidszorg" (COTG) codes (unilateral inguinal hernia repair: CTG335700; bilateral inguinal hernia repair: CTG335701; incarcerated inguinal hernia repair without bowel resection: CTG335702; incarcerated inguinal hernia repair with bowel resection: CTG334639; recurrent inguinal hernia repair: CTG335710).

\section{Study groups and patient exclusion criteria}

Two groups of premature infants were distinguished: (1) those who underwent elective inguinal hernia repair and (2) those who had to undergo an emergency procedure because of incarceration of contents in the hernia sac. Emergency surgery was defined as inguinal hernia repair performed within $24 \mathrm{~h}$ after incarceration and not scheduled on the surgeon's elective procedure list. Patient characteristics and clinical data were collected from medical records and have been published already [6]. Premature infants who were initially admitted for conditions other than inguinal hernia, who were still hospitalized since birth, or who were admitted more than $72 \mathrm{~h}$ before surgery, were excluded because it was not possible to separate costs associated with inguinal hernia from costs associated with treatment for other conditions. All patients were operated on after discharge from their birth hospitalization; in all prematures general anesthesia were used, whether or not combined with a caudal block or loco-regional anesthesia. Premature born infants operated on with a gestational age of 40 weeks or younger, were postoperatively admitted to the NICU. Patients with a gestational age of 40-45 weeks had to stay in the recovery room for $2 \mathrm{~h}$ after which they were admitted to the ward. This institutional policy is based on national guidelines from the Dutch Society of Anaesthesia. Prolonged mechanical ventilation was only performed if clinically indicated, mostly due to pulmonary comorbidities.

\section{Cost calculation}

Direct medical costs were considered to be all costs within the hospital sector directly related to the studied treatment, including the hospital's outpatient setting. Per patient, we took into account (1) costs of hospitalization (NICU or high care or medium care unit, and readmissions); (2) costs of outpatient care (i.e., visits to $\mathrm{OPC}$, visits to $\mathrm{ED}$, and telephone consultations); (3) costs of diagnostic procedures and perioperative care (i.e., ultrasound and anaesthesiologist consultations); and (4) costs of surgical procedures (i.e., operating rooms (OR), OR during after hours, 
Table 1 Cost categories and data used in cost calculation based on real costs

\begin{tabular}{llc}
\hline & Parameter & Cost price $(€)$ \\
\hline Hospitalization & & \\
NICU & Day & 1540 \\
High care unit & Night & 859 \\
Normal care unit & Night & 447 \\
Outpatient clinic visits & & \\
First visit to OPC & Visit & 226 \\
Follow-up at OPC & Visit & 175 \\
Emergency department & Visit & 243 \\
Telephonic consultation & Call & 135 \\
Perioperative consultation & & \\
Ultrasound & Number & 81 \\
Preoperative consultation ANA & Visit & 125 \\
Surgical procedure & & \\
Use of OR & $/ 10$ min & 74 \\
Use of OR afterhours & $/ 10$ min & 223 \\
Anesthetist's fee & $/ 10$ min & 22 \\
Surgeon's fee & $/ 10$ min & 22 \\
Anesthetist's fee afterhours & $/ 10$ min & 22 \\
Surgeon's fee afterhours & $/ 10$ min & 22 \\
\hline
\end{tabular}

All cost prices are based on real costs rather than charges. Costs in Euro for the first of January 2014, when 1 Euro $(€)$ equalled approximately 1.38 US Dollar

NICU neonatal intensive care unit, OPC outpatient clinic, ANA anaesthesiology, $O R$ operating room

Day, cost price calculated for each calendar day a patient is hospitalized; night, cost price calculated for every overnight stay per patient

reoperations and costs of anaesthesiologists and surgeons). Table 1 provides an overview of the cost categories and the unit prices used in the cost calculations.

Real medical costs were calculated by multiplying the volumes of healthcare use with the corresponding unit prices. Unit prices were determined with the micro-costing method, which implies a detailed inventory and measurement of all resources used [12, 13]. For hospital days, the cost price included costs for nursing staff, buildings, and equipment (specialists' fees not included). The unit price for the NICU was calculated for each calendar day an individual was hospitalized; the unit price for a stay on the medium- or high-care was calculated per overnight stay. Regarding the costs of the surgical procedure, the cost price included fees of the surgeons and anesthesiologists, costs of the OR, including costs of OR assistants, anesthesiology nurses, equipment, buildings, etc. For visits to the ED and OPC, preoperative consultation, and ultrasounds, the cost price included the fees of the surgeons, anesthesiologists, and radiologists. All admissions due to a reoperation or admissions that were inguinal hernia related, or related to the first admission, were interpret to be readmissions. We reported costs in Euro for the first of January 2014, when 1 Euro $(€)$ equalled approximately 1.38 US Dollar.

\section{Data analysis}

SPSS 21.0 (SPSS Inc., Chicago, IL, USA) was used for all statistical analyses. Fisher exact tests and Mann-Whitney $U$ tests were used to compare costs of emergency- and elective repair. Since cost data per patient are typically highly skewed, we used nonparametric simple bootstrap techniques with a 1000 samples. To derive a $95 \%$ confidence interval for the differences in distributions of the direct medical costs, we used the percentile bootstrap method. We used a linear regression model to control for confounders. Potential risk factors with a $p$ value $<0.05$ were included in the model (i.e., birth weight, age at repair, history of bradycardia, and mechanical ventilation). A $p$ value of $<0.05$ was considered statistically significant.

\section{Results}

Between January 2010 and December 2013, 142 premature infants underwent inguinal repair within 3 months after birth. Ten patients were excluded because they were admitted more than $72 \mathrm{~h}$ before surgery. The mean gestational age at birth was 34 weeks (SD 17 days). Mean birth weight was $1916 \mathrm{~g}$ (SD $543 \mathrm{~g}$ ), mean follow-up was 27 weeks (SD 15 weeks), and mean time from diagnosis to surgery was 13 days (SD 12 days).

Seventy-two of the 132 included infants (55\%) presented with a symptomatic inguinal hernia, of whom 38 (29\%) needed subsequent emergency repair due to incarceration within $24 \mathrm{~h}$. In the other 34 infants $(26 \%)$, the symptomatic hernias could be reduced manually and delayed elective surgery was performed. Nine patients had no incarceration at first presentation, but returned to the ED with an incarcerated hernia and had to undergo emergency surgery. Table 2 presents characteristics of the two groups. Mean birth weight of patients who needed emergency repair was significantly lower than that of patients who underwent elective repair (1738 vs. $2001 \mathrm{~g}$ ). Mean post conception age at the time of repair was 41 weeks (SD 3.2 weeks) in the emergency group and 44 weeks (SD 2.8 weeks) in the elective repair group $(p<0.001)$. Mean age at diagnosis was 53 days (SD 18 days) versus 53 days (SD 14 days; $p=0.805$ ), respectively. Mean time between first presentation and repair was 18.0 days (SD 10.7 days) for elective repair and 1.8 days (SD 2.9 days) for emergency repair $(p<0.001)$. Mean weight at repair was 
Table 2 Baseline characteristics

\begin{tabular}{lccc}
\hline & Elective repair $(N=94)$ & Emergency repair $(N=38)$ & $p$ value \\
\hline Male gender $(\%)$ & $78(83)$ & $35(92)$ & 0.273 \\
Birth weight [g (SD)] & $2001(552)$ & $1738(483)$ & $0.022^{* *}$ \\
Gestational age at repair [weeks (SD)] & $44(2.8)$ & $41(3.2)$ & $4(11)$ \\
Cardiac anomalies (\%) & $7(8)$ & $8(23)$ & $0.001^{* *}$ \\
Bradycardia of prematurity (\%) & $7(8)$ & $14(40)$ & 0.727 \\
Apnoea of prematurity (\%) & $20(23)$ & $10(29)$ & $4(11)$ \\
IRDS (\%) & $13(15)$ & $18(51)$ & $0.074^{*}$ \\
BPD (\%) & $2(4)$ & $4(11)$ & 0.123 \\
History of mechanical ventilation $(\%)$ & $26(30)$ & $2(6)$ & 0.056 \\
GERD (\%) & $5(6)$ & $2(2)$ & $0.036^{*}$ \\
NEC (\%) & 0.276 \\
\hline
\end{tabular}

All values presented as percentages or means (standard deviations), $p$ values are two-sided

IRDS infant respiratory distress syndrome, $B P D$ bronchopulmonary dysplasia, GERD gastroesophageal reflux disease, NEC necrotising enterocolitis

* Statistically significant (Fisher exact test)

** Statistically significant (Mann-Whitney $U$ test)

$3912 \mathrm{~g} \mathrm{(SD} 865 \mathrm{~g}$ ) for elective repair and 3206 (SD $769 \mathrm{~g}$ ) for the emergency repair $(p<0.001)$.

After elective surgery 48 out of $94(51 \%)$ patients were admitted to the NICU and after emergency repair 37 out of $38(97 \% ; p<0.001)$. Seven $(18 \%)$ patients needed prolonged mechanical ventilation at the NICU after emergency repair versus two (2\%) after elective repair $(p=0.002)$. The median duration of prolonged mechanical ventilation was $123 \mathrm{~min}$ (range 31; $2379 \mathrm{~min}$ ). The recurrence rate after an emergency procedure was significantly higher than that after elective repair (13 vs. $2 \%$; $p=0.021)$.

\section{Cost analysis}

Based on the cost prices outlined in Table 1, the mean total costs per patient amounted to $€ 5477$ (SD $€ 3280$ ). Contributions of four different cost components to the total costs are outlined in Fig. 1. At mean $€ 3573$ (SD $€ 3280$ ), cost of

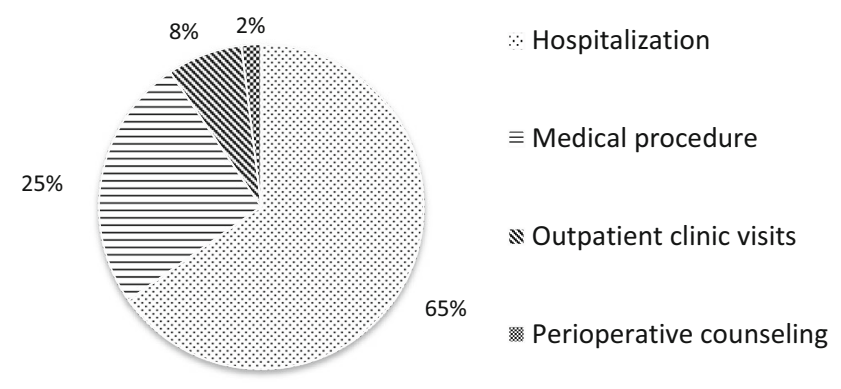

Fig. 1 Costs divided into four subcategories presented with percentages hospitalizations made up two thirds of the total costs. The mean costs of the surgical procedures were $€ 1385$ (SD $€ 1181$ ), of perioperative consultation $€ 107$ (SD €92), and of OPC and/or ED visits €412 (SD €302).

Emergency repair resulted in 1.58 times higher total costs compared with elective repair: €7418 (SD €4484) versus $€ 4693$ ( $S D € 3465 ; p<0.001$ ), respectively. These are further detailed in Table 3. Except for the costs of perioperative consultations, costs for all categories were higher on average in the case of emergency repair. The difference in costs of hospitalizations $(€ 1501)$ accounted for more than half of the difference in total costs between the two patient groups (€2725).

When we controlled for possible confounding variables in the linear regression analysis (i.e., birth weight, age at repair, history of bradycardia, mechanical ventilation and emergency repair), the difference in total costs between emergency and elective repair remained $€ 1183$ (95\% CI $-1196 ; 3044)$ in favor of elective repair. None of the variables included in the linear regression analysis were found to be contributing significantly to the total costs.

\section{Discussion}

Insight into the actual costs of different strategies in inguinal hernia repair would be relevant for planning and organization of care around premature infants with inguinal hernia. The present study is the first study that provides information about the true medical costs of inguinal hernia repair in premature infants, comparing elective and emergency repair. Emergency repair appeared to be associated with significantly higher direct medical costs. 
Table 3 Difference in costs per patient between emergency and elective repair

\begin{tabular}{|c|c|c|c|}
\hline & $\begin{array}{l}\text { Elective repair } \\
(\mathrm{N}=94)\end{array}$ & $\begin{array}{c}\text { Emergency } \\
\text { repair } \\
(\mathrm{N}=\mathbf{3 8})\end{array}$ & $\begin{array}{l}\text { Difference } \\
\text { bootstrap } \\
(95 \% \text { CI })\end{array}$ \\
\hline Pre-op high care- and medium care, $€(\mathrm{SD})$ & $164(459)$ & $271(405)$ & $107(-52 ; 268)$ \\
\hline NICU, $€(\mathrm{SD})$ & $2491(1154)$ & $3608(1400)$ & $1117(643 ; 1651)$ \\
\hline Post-op high- and medium care, $€(\mathrm{SD})$ & $328(2415)$ & $27(167)$ & $-301(-872 ; 37)$ \\
\hline Readmissions ward, $€(\mathrm{SD})$ & $91(408)$ & $452(2339)$ & $361(-108 ; 1307)$ \\
\hline Readmissions NICU, $€(\mathrm{SD})$ & $66(447)$ & $284(1004)$ & $218(-62 ; 591)$ \\
\hline Hospitalization & 3141 (2986) & $4642(3746)$ & $1501(283 ; 3050)$ \\
\hline Emergency department, $€$ (SD) & $136(217)$ & $274(233)$ & $138(53 ; 222)$ \\
\hline OPC first visits, $€(\mathrm{SD})$ & $180(103)$ & $113(137)$ & $-67(-114 ;-18)$ \\
\hline OPC follow up visits, $€(\mathrm{SD})$ & $52(135)$ & $69(155)$ & $17(-37 ; 77)$ \\
\hline Telephonic consultation, $€$ (SD) & $19(61)$ & $28(64)$ & $10(14 ; 34)$ \\
\hline Outpatient clinic visits & $388(264)$ & $485(369)$ & $66(-35 ; 228)$ \\
\hline Ultrasound, $€(\mathrm{SD})$ & $8(34)$ & $28(47)$ & $20(3 ; 38)$ \\
\hline Preop. consultation anesthetist, $€(\mathrm{SD})$ & $114(63)$ & $43(73)$ & $-72(-97 ;-47)$ \\
\hline Perioperative consultation & $122(84)$ & $71(100)$ & $-52(-85 ;-15)$ \\
\hline Operating room, $€(\mathrm{SD})$ & $562(211)$ & $686(270)$ & $124(32 ; 230)$ \\
\hline Operating room afterhours, $€(\mathrm{SD})$ & - & $746(1037)$ & $746(435 ; 1071)$ \\
\hline Reoperation, $€(\mathrm{SD})$ & $143(727)$ & $231(589)$ & $87(-153 ; 336)$ \\
\hline Surgeon, $€(\mathrm{SD})$ & $170(64)$ & $207(82)$ & $37(10 ; 69)$ \\
\hline Surgeon afterhours, $€(\mathrm{SD})$ & - & $75(104)$ & $75(44 ; 109)$ \\
\hline Anesthetist, $€(\mathrm{SD})$ & $170(64)$ & $207(82)$ & $37(10 ; 69)$ \\
\hline Anesthetist afterhours, $€$ (SD) & - & $75(104)$ & $75(44 ; 109)$ \\
\hline Surgical procedures & $1045(886)$ & $2227(1423)$ & $1182(754 ; 1660)$ \\
\hline Total costs, $€(\mathrm{SD})$ & $4695(3463)$ & $7424(4480)$ & $2729(1291 ; 4166)$ \\
\hline
\end{tabular}

Costs in Euro for the first of January 2014, when 1 Euro $(€)$ equalled approximately 1.38 US Dollar. All costs are rounded to whole Euro's. Nonparametric bootstrap techniques to derive a $95 \%$ confidence interval were used CI is presented with lower and upper values between brackets

The values presented in bold are subcategories containing the sum of the aforementioned values

$S D$ standard deviation, NICU neonatal intensive care unit, $O P C$ outpatient clinic, $C I$ confidence interval

Hospitalization was accountable for approximately two thirds of the total costs. Therefore, reducing length of stay on the ward and NICU could be an important target for cost reduction. The mean costs of hospitalization after elective repair were lower compared to costs after emergency repair. Almost all premature infants who had to undergo emergency repair were postoperatively admitted to the NICU and immediately discharged after overnight observation without admission to the high- or medium care unit. The question arises whether all patients should be admitted to the NICU postoperatively after an emergency procedure, or whether it would be possible to select only those who really need close observation. Very-low-birth weight premature infants with severe comorbidities are more likely to develop postoperative respiratory and cardiac incidents; obviously, a close observation or intervention on the NICU is advised for those [14-16]. The incarceration rate in our study population is relatively high compared to literature $[2,4,17]$. This can be explained by the fact that our hospital is the only tertiary academic pediatric hospital in the region and covers 4.5 million inhabitants. Our hospital is the only hospital in its region that is allowed to perform surgery in premature infants. Additionally, it also explains the relatively high recurrence rate because all patients with recurrences hernia will always return to our institution.

There were significant differences between the two study groups in pulmonary comorbidities, birth weight, weight at repair, age at repair, age at diagnosis and prolonged mechanical ventilation, which could be explained by the longer hospitalization and NICU stays in patients after emergency surgery. Still, preventing these patients from emergency repair could result in a reduction of costs. 
Only one other study made a cost analysis of inguinal hernia in premature infants [7]. This study compared premature infants with full-term born infants, however, these groups are hardly comparable in terms of comorbidity, risk factors, and complications. In this study all premature patients underwent surgery with the use of general anesthesia. However, the use of spinal anesthesia alone can be advocated in patients with pre-existing diseases and incarceration undergoing inguinal hernia repair [18]. The costs of preoperative consultation of anaesthesiologist were significantly higher in the elective repair group. If patients were scheduled for elective repair, it was common practice in our hospital to consult an anaesthesiologist; in case of emergency surgery this was omitted.

The health- and peri-operative care around premature born infants have dramatically changed over the last three decades and new technical advances in neonatal surgery have driven up health care costs [10]. The policy to perform surgery during birth hospitalization before discharge from the NICU is still relevant, as this could prevent these neonates from readmissions and incarceration resulting in emergency surgery. Early herniotomy has already shown to improve postoperative outcomes without increasing postoperative bradycardia, apnea and other events [5]. Nevertheless, repair of inguinal hernia before discharge from the NICU is associated with a prolonged hospital stay [4]. Sufficient capacity, resources and a decision-making tool enabling surgery at least 1 week before planned discharge could solve this problem. Early surgery, on the other hand, is associated with a higher risk of surgical and anesthetic complications in view of the infant's lower body weight. The present study has limitations. Selection bias could have occurred due to the absence of a protocol on timing of inguinal hernia repair in premature infants. Furthermore, a financial analysis is dependant on the local financing process, and local institutional policies (e.g., in our institution, specialists' fees are similar during day and night shifts and admission to the NICU postoperative for patients under the gestational age of 40 weeks).

Keeping this limitation in mind, we provide solid evidence on the direct medical costs of emergency repair in premature infants. It appeared that emergency repair is almost $60 \%$ more expensive than elective repair. Therefore, it can be argued that preventing premature infants from incarceration, for which often emergency repair is needed, will reduce aggregate direct medical costs. Further evidence on actual costs of different strategies in inguinal hernia repair in premature infants would be welcome as a basis for the development of decision-making tools. For instance, it would be worth knowing the additional costs of performing surgery during the birth hospitalization compared to the costs of elective surgery when delayed. Outcomes of new prospective randomized trials, including cost-effectiveness, will help allocate health care resources even more efficiently.

Acknowledgments The authors thank Ko Hagoort for editorial advice.

\section{Compliance with ethical standards}

Conflict of interest The authors of this manuscript have no conflicts of interest to disclose.

Open Access This article is distributed under the terms of the Creative Commons Attribution-NonCommercial 4.0 International License (http://creativecommons.org/licenses/by-nc/4.0/), which permits any noncommercial use, distribution, and reproduction in any medium, provided you give appropriate credit to the original author(s) and the source, provide a link to the Creative Commons license, and indicate if changes were made.

\section{References}

1. Pan ML, Chang WP, Lee HC, Tsai HL, Liu CS, Liou DM, Sung YJ, Chin TW (2013) A longitudinal cohort study of incidence rates of inguinal hernia repair in 0 - to 6-year-old children. J Pediatr Surg 48:2327-2331

2. Lautz TB, Raval MV, Reynolds M (2011) Does timing matter? Premature neonates with inguinal hernia. J Pediatr 158:573-577

3. Lau ST, Lee YH, Caty MG (2007) Current management of hernias and hydroceles. Semin Pediatr Surg 16:50-57

4. Lee SL, Gleason JM, Sydorak RM (2011) A critical review of premature infants with inguinal hernias: optimal timing of repair, incarceration risk, and postoperative apnea. J Pediatr Surg 46:217-220

5. Vaos G, Gardikis S, Kambouri K, Sigalas I, Kourakis G, Petoussis G (2010) Optimal timing for repair of an inguinal hernia in premature infants. Pediatr Surg Int 26:379-385

6. de Goede B, Verhelst J, van Kempen BJ, Baartmans MG, Langeveld HR, Halm JA, Kazemier G, Lange JF, Wijnen RM (2015) Very low birth weight is an independent risk factor for emergency surgery in premature infants with inguinal hernia. J Am Coll Surg 220:347-352

7. Groff DB, Nagaraj HS, Pietsch JB (1985) Inguinal hernias in premature infants operated on before discharge from the neonatal intensive care unit. Arch Surg 120:962-963

8. Melone JH, Schwartz MZ, Tyson KRT, Marr CC, Greenholz SK, Taub JE, Hough VJ (1992) Outpatient inguinal herniorrhaphy in premature infants: is it safe? J Pediatr Surg 27:203-208

9. Peutrell JM, Hughes DG (1992) Ex-premature infants can safely have outpatient inguinal herniotomies. J Pediatr Surg 27:1487-1488

10. Poley MJ, Brouwer WB, Busschbach JJ, Hazebroek FW, Tibboel D, Rutten FF, Molenaar JC (2008) Cost-effectiveness of neonatal surgery: first greeted with scepticism, now increasingly accepted. Pediatr Surg Int 24:119-127

11. Stolk EA, Post HA, Rutten FF, Molenaar JC, Busschbach JJ (2000) Cost-effectiveness of neonatal surgery: a review. J Pediatr Surg 35:588-592

12. Drummond MF, O'Brien BJ, Stoddart GL, Torrance GW (1997) Torrance Methods for the economic evaluation of health care programmes. Oxford University Press, Oxford

13. Gold M, Siegel JE, Russel LB, Weinstein MC (1996) Cost-effectiveness in health and medicine. Oxford University Press, New York 
14. Kinouchi K (2004) Anaesthetic considerations for the management of very low and extremely low birth weight infants. Best Pract Res Clin Anaesthesiol 18:273-290

15. Holzman RS (1994) Morbidity and mortality in pediatric anesthesia. Pediatr Clin N Am 41:239-256

16. Rappaport B, Mellon RD, Simone A, Woodcock J (2011) Defining safe use of anesthesia in children. N Engl J Med 364:1387-1390
17. Baird R, Gholoum S, Laberge JM, Puligandla P (2011) Prematurity, not age at operation or incarceration, impacts complication rates of inguinal hernia repair. J Pediatr Surg 46:908-911

18. Lambertz A, Schalte G, Winter J, Roth A, Busch D, Ulmer TF, Steinau G, Neumann UP, Klink CD (2014) Spinal anesthesia for inguinal hernia repair in infants: a feasible and safe method even in emergency cases. Pediatr Surg Int 30:1069-1073 